\title{
Cama-de-frango em mono e policultivo de fáfia com cravo-de-defunto e manjericão
}

\author{
Valdenise C Barboza ${ }^{2}$; Maria do Carmo Vieira ${ }^{2,3}$; Néstor A Heredia Zárate ${ }^{2,3}$; Victor Hugo Padovezzi ${ }^{4}$; \\ Maicom Jorge G dos Santos ${ }^{4}$ \\ ${ }^{2}$ UFGD, C. Postal 533, 79804-970 Dourados-MS; ${ }^{3}$ Bolsistas CNPq; ${ }^{4}$ Alunos, Curso de Agronomia, Faculdades Anhanguera de Dourados; \\ valcarbonari@hotmail.com; mariavieira@ufgd.edu.br
}

\section{RESUMO}

O experimento foi conduzido em campo da Universidade Federal da Grande Dourados (UFGD), em Dourados-MS, de março de 2005 a setembro de 2006, em Latossolo Vermelho distroférrico. O objetivo foi avaliar a produção da Pfaffia glomerata (Spreng) Pedersen (fáfia) em monocultivo e os policultivos com Tagetes erecta L. (cravo-de-defunto) e Ocimum basilicum L. (manjericão), sem e com incorporação de cama-de-frango semidecomposta. Os fatores em estudo foram a fáfia (F), o cravo-de-defunto (C) e o manjericão (M) em monocultivos e os policultivos com duas fileiras de fáfia e três de cravo $\left(\mathrm{F}_{2} \mathrm{C}_{3}\right)$ e duas fileiras de fáfia e três de manjericão $\left(\mathrm{F}_{2} \mathrm{M}_{3}\right)$, todos sem e com incorporação ao solo de cama-de-frango de corte semidecomposta Os dez tratamentos foram arranjados no delineamento blocos casualizados, com quatro repetições. A produção de massa fresca e seca da parte aérea da fáfia foi maior $\left(13,22 \mathrm{t} \mathrm{ha}^{-1} \mathrm{e} 4,39 \mathrm{tha}^{-1}\right.$, respectivamente) em monocultivo, independente do uso da cama-defrango. Por outro lado, nenhum dos tratamentos influenciou a massa fresca e seca e o número de raízes da fáfia, que foram, em média, de 10,02 e 2,07 tha ${ }^{-1}$ e 417.916 raízes ha ${ }^{-1}$, respectivamente. O diâmetro das raízes foi maior $(23,5 \mathrm{~mm})$ no policultivo com o manjericão. As produções de massas frescas e secas dos capítulos florais do cravode-defunto foram maiores $\left(14,28 \mathrm{tha}^{-1} \mathrm{e} 1,278 \mathrm{tha}^{-1}\right.$, respectivamente) no policultivo com a fáfia, mas apenas a produção de massa fresca dos capítulos foi maior $\left(14,17 \mathrm{t} \mathrm{ha}^{-1}\right)$ com o uso da cama-de-frango. A produção de parte aérea do manjericão foram maiores $(52,91 \mathrm{t}$ $\mathrm{ha}^{-1}$ ) no policultivo, independente com qual espécie; porém, não foi influenciada pelo uso da cama-de-frango. A razão de área equivalente (RAE) para o policultivo da fáfia com o cravo-de-defunto foi de 2,15 com cama-de-frango e de 1,99 sem cama e com o manjericão, foi 2,44 com cama e de 3,08 sem cama. Como os valores foram maiores que 1,0 , indicam que os policultivos foram efetivos.

Palavras-chave: Pfaffia glomerata, Tagetes erecta, Ocimum basilicum, associação de culturas.

\begin{abstract}
Poultry manure in mono and intercrop of Brazilian ginseng with marigold and basil

This study was carried out in field of the Universidade Federal da Grande Dourados, in Dourados, Brazil, during the period from March 2005 to September 2006. The aim of the experiment was to evaluate Pfaffia glomerata (Spreng) Pedersen yield under monocropping system or intercropped with Tagetes erecta L. and Ocimum basilicum L, in a Distroferric Red Latosol (sand loam Rhodic Oxisol), using semi-decomposed poultry manure (PM). The study objects were Brazilian ginseng (BG), marigold (M) and basil (B) under monocropping and the intercropping of two Brazilian ginseng, three marigold $\left(\mathrm{BG}_{2} \mathrm{M}_{3}\right)$ and three basil $\left(\mathrm{BG}_{2} \mathrm{~B}_{3}\right)$ lines, all of them with or without semi-decomposed poultry manure (PM) as fertilizer. Ten treatments were arranged in randomized blocks design, with four replications. Fresh and dry weight production from shoot of Brazilian ginseng were higher (13.22 $\mathrm{t} \mathrm{ha}^{-1}$ and $4.39 \mathrm{t} \mathrm{ha}^{-1}$, respectively) under monocropping, independently of the use of poultry manure. Nevertheless, none of experimental designs influenced the dry and fresh weight production or root number of Brazilian ginseng which produced average values of 10.02 and $2.07 \mathrm{t} \mathrm{ha}^{-1}$ and 417,916 roots $\mathrm{ha}^{-1}$, respectively. Root diameter was higher $(23.5 \mathrm{~mm})$ under intercropping system with basil. Dry and fresh weight of marigold flowers were higher (14.28 $\mathrm{tha}^{-1}$ and $1.278 \mathrm{t} \mathrm{ha}^{-1}$, respectively) when intercropped with Brazilian ginseng, but only fresh weight of the flowers was increased $\left(14.17 \mathrm{tha}^{-1}\right)$ by poultry manure application. Basil shoot production was higher (52.91 tha-1) when intercropped, independently of the used species; however, they were not influenced by the use of poultry manure. Land equivalent ration (LER) for the Brazilian ginseng intercropped with marigold was 2.15 under poultry manure application and 1.99 without it, and for the basil 2.44 under poultry manure application and 3.08 without it. Values higher than 1.0 indicate that the intercropping systems were effective.
\end{abstract}

Keywords: Pfaffia glomerata, Tagetes erecta, Ocimum basilicum, intercrops.

(Recebido para publicação em 28 de novembro de 2008; aceito em 17 de agosto de 2010) (Received on November 28, 2008; accepted on August 17, 2010)

\begin{abstract}
A Pfaffia glomerata (Spreng) Pedersen (fáfia), Amaranthaceae, é uma espécie de clima tropical, embora possa adaptar-se ao subtropical. Ocorre em todo o Brasil e países limítrofes, com precipitação pluviométrica
\end{abstract}

entre 1200 a $1500 \mathrm{~mm}$ anuais e altitudes de até $1000 \mathrm{~m}$. É frequente no Cerrado e outros biomas do Mato Grosso do Sul, incluindo o Pantanal, nas margens e ilhas do Rio Paraná, Paranapanema e Ivaí, entre São Paulo, Mato Grosso do
Sul e Paraná. Está adaptada aos ciclos de cheia e seca dessas áreas, apresentando plasticidade às mudanças ambientais, que podem facilitar sua exploração e cultivo. Por isso, é natural o grande número de formas e variedades dentro

${ }^{1}$ Parte da tese de doutorado da primeira autora, apresentada ao Programa de Pós-Graduação em Agronomia-UFGD. 
da espécie, pela ampla distribuição geográfica, ocupando condições edafoclimáticas distintas (Corrêa Júnior \& Ming, 2001; Corrêa Júnior \& Ming, 2004; Kamada et al., 2009).

A importância medicinal e econômica da fáfia, na forma de fitomedicamentos e suplementos alimentares, é demonstrada pela ampla industrialização e comercialização de seus produtos, que são encontrados na maioria das farmácias e drogarias brasileiras, o que tornou-se ameaça às populações naturais devido ao uso das raízes e, principalmente, devido ao extrativismo. Dentre os vários usos populares das raízes da fáfia, alguns têm sido referendados cientificamente, dentre eles, proteção da mucosa gástrica; atividade tônica, afrodisíaca, antinociceptiva, antinflamatória e adaptógena, contra estresses físico e mental (Russowski \& Nicoloso, 2003; Freitas et al., 2004; Marques et al., 2004; Neto et al., 2005; Otofuji et al., 2005; Mendes \& Carlini, 2007; Freitas et al., 2008; Freitas et al., 2009).

A produtividade da fáfia é maior em substratos à base de cama-de-frango e terriço, na proporção de $1: 1$, por favorecerem a formação de maior número e tamanho de raízes, mais compridas, embora com menor diâmetro. Plantas cultivadas na mistura de esterco de aves e terriço, na proporção de 1:5 e 1:3, produziram 3,7 e 3,5 vezes mais massa seca, respectivamente, do que as plantas não cultivadas com esterco (Bentes et al., 2000). Nascimento et al. (2007) avaliaram a produção da fáfia e da tansagem, em mono e policultivo e verificaram que a razão de área equivalente (RAE) foi de 1,07, para o policultivo de três fileiras de tansagem alternadas com duas fileiras de fáfia $\left(\mathrm{T}_{3} \mathrm{~F}_{2}\right)$ espaçadas de 36 $\mathrm{cm}$ considerando as produtividades de massa fresca das folhas de tansagem e das raízes de fáfia [RAE $\mathrm{T}_{3} \mathrm{~F}_{2}(36)=$ $1,07]$. A RAE para o policultivo quatro fileiras de tansagem alternadas com duas fileiras de fáfia $\left(\mathrm{T}_{4} \mathrm{~F}_{2}\right)$ espaçadas de 54 $\mathrm{cm}$ foi de 1,50, indicando, nos dois casos, a viabilidade do policultivo.

A intensa exploração predatória das reservas naturais justifica a elaboração de planos de manejo ou projetos de cultivo para a fáfia. Dentre eles, poderia ser utilizado o policultivo de espécies, prática tradicional de produção de alimentos e biomassa nas regiões tropicais, onde pequenas propriedades e operações intensivas predominam. A fáfia tem ciclo de cultivo relativamente longo, podendo as raízes ser colhidas a partir do primeiro até o quinto ano após o plantio (Corrêa Júnior \& Ming, 2004) e considerando que nos primeiros meses o solo fica descoberto em grande extensão, pode ser estudada a possibilidade de policultivo dessa espécie com outras que cobrem mais rapidamente o solo, dentre elas Tagetes erecta (cravo-de-defunto) e Ocimum basilicum (manjericão).

Espécies de Tagetes sp., conhecidas como "flor dos mortos" e cravo-de-defunto, possuem óleos essenciais usados como condimento, corante de alimentos, inseticida, fungicida, para controlar ervas daninhas e como fonte de pigmento para ração de galinhas, visando intensificar a cor amarela das gemas dos ovos. Têm sido usadas para controlar fitonematóides e a maioria dos estudos indica que essas plantas são muito eficientes, especialmente, contra as espécies de Pratylenchus e Meloidogyne (Medina et al., 1993; Gutiérrez et al., 2006). Em melão, T. patula serviu como planta atrativa para Neohydatothrips sp.; Frankliniella sp. 1; Caliothrips sp. e F. schultzei (Thysanoptera) e, dessa forma, pode ser recomendado para plantio na bordadura do cultivo principal para reduzir a infestação por espécies fitófagas de tripes (Peres et al., 2009).

Tagetes são usadas em rotação de culturas, mas, em muitas situações, funcionam muito bem em policultivo. A produção do tomate em policultivo com Tagetes erecta foi maior do que no monocultivo, devido ao aumento da taxa fotossintética líquida, maior teor de clorofila, maior espessura das folhas e em consequência, maior número de cachos e de frutos (Gómez-Rodriguez, 2007).

O manjericão pode ser utilizado como planta medicinal, aromática ou condimentar in natura e processado, como folhas secas inteiras ou moídas como fonte de óleo essencial valorizado no mercado internacional pelo alto teor de linalol. O óleo essencial, obtido da destilação de folhas e inflorescências, é utilizado na culinária e na aromatização de alimentos e bebida, além da indústria de cosméticos. Apresenta propriedades inseticidas, repelentes e antimicrobianas, daí seu uso na conservação de grãos (Fernandes et al., 2004; Carvalho Filho et al., 2006).

Luz et al. (2009) utilizaram camade-frango nas doses 2, 4, 6, 8 e $10 \mathrm{~kg}$ $\mathrm{m}^{-2}$ em dois genótipos de manjericão e não observaram influência significativa nas alturas das plantas, no comprimento e largura das folhas nem no teor de óleo essencial. Por outro lado, houve influência no teor de linalol do óleo essencial dos dois genótipos estudados. Blank et al. (2005) estudaram cinco tipos de adubação (adubo formulado Hortosafra ${ }^{\circledR}$, esterco de galinha, esterco de galinha + Hortosafra $^{\circledR}$, esterco bovino, esterco bovino + Hortosafra $\left.^{\circledR}\right)$ e três horários de colheita $(8: 00,12: 00$ e 16:00 h) na produção de Ocimum basilicum L. cv. Genovese. A massa seca da parte aérea das plantas foi significativamente maior quando foram adubadas com Hortosafra $^{\circledR}$ e esterco de galinha, em relação ao esterco bovino. O horário de colheita das plantas não influenciou significativamente o teor e rendimento de óleo essencial.

Nenhum trabalho foi encontrado na bibliografia consultada com policultivo de fáfia com Tagetes e, ou com manjericão. Por isso, objetivou-se com a realização deste trabalho avaliar o crescimento e a produção da Pfaffia glomerata em mono e policultivo com Tagetes erecta e Ocimum basilicum, cultivados em solo sem e com cama-de-frango.

\section{MATERIAL E MÉTODOS}

O experimento foi desenvolvido em campo da Universidade Federal da Grande Dourados em Dourados-MS de março de 2005 a setembro de 2006. Situa-se em latitude de $22^{\circ} 1143$ "S, longitude de $54^{\circ} 5608^{\prime \prime} \mathrm{W}$ e altitude de 458 m. O clima da região, segundo a classificação de Köppen, é Mesotérmico Úmido; do tipo Cwa, com temperaturas e precipitações médias anuais variando de 20 a $24^{\circ} \mathrm{C}$ e de $1250 \mathrm{~mm}$ a $1500 \mathrm{~mm}$, respectivamente.

O solo é do tipo Latossolo Vermelho Distroférrico, de textura argilosa, com 
as seguintes características químicas, na profundidade de $0-20 \mathrm{~cm}$ : $\mathrm{pH}$ em água $=5,1 ; \mathrm{pH}$ em $\mathrm{CaCl}=4,5 ;$ M.O. $=31,2$ $\mathrm{g} \mathrm{kg}^{-1} ; \mathrm{P}=24 \mathrm{mg} \mathrm{dm}^{-3}$; e 5,0; 6,9; 22,9; 16,$3 ; 72,0 ; 34,0$ e $106,10 \mathrm{mmol} \mathrm{dm}^{-3} \mathrm{de}$ $\mathrm{K}, \mathrm{Al}, \mathrm{Ca}$ e $\mathrm{Mg}, \mathrm{H}+\mathrm{Al}$, SB e CTC, respectivamente. Os resultados das análises físicas do solo foram 760,0; 90,9; 89,2 e $60,2 \mathrm{~g} \mathrm{~kg}^{-1}$ de argila, silte, areia grossa e areia fina, respectivamente; densidade do solo $=1,12$ e densidade de partículas $=2,08$. A cama-de-frango apresentou $\mathrm{C}$ orgânico = 20,56 $\mathrm{g} \mathrm{kg}^{-1}$ e 28,50; 24,30; $1,87 \mathrm{~g} \mathrm{~kg}^{-1}$ de $\mathrm{P}, \mathrm{K}$ e N, respectivamente, além de relação $\mathrm{C} / \mathrm{N}=10,99$.

Os fatores em estudo foram a fáfia (Pfaffia glomerata) (F), o cravo-dedefunto (Tagetes erecta) (C) e o manjericão (Ocimum basilicum) (M) em monocultivos e os policultivos com duas fileiras de fáfia e três de cravode-defunto $\left(\mathrm{F}_{2} \mathrm{C}_{3}\right)$ e duas fileiras de fáfia e três de manjericão $\left(\mathrm{F}_{2} \mathrm{M}_{3}\right)$, todos sem e com cama-de-frango de corte semidecomposta incorporada ao solo. Os dez tratamentos foram arranjados no delineamento experimental blocos casualizados, com quatro repetições. A área total de cada parcela foi de 4,5 $\mathrm{m}^{2}$ (1,5 m de largura $\mathrm{x} 3,0 \mathrm{~m}$ de comprimento) e a área útil de $3,0 \mathrm{~m}^{2}$ (1,0 m de largura do canteiro x 3,0 m de comprimento). As sementes da fáfia foram obtidas de plantas cultivadas no Horto de Plantas Medicinais da UFGD, com dois anos de idade; cuja origem foi de sementes coletadas de várias plantas da área nativa em Dourados. O manjericão de folha miúda e o cravo-de-defunto são cultivares comerciais adquiridos da Feltrin.

As mudas das três espécies em estudo foram produzidas em bandejas de poliestireno expandido de 128 células, utilizando-se o substrato comercial Plantmax ${ }^{\circledR}$. O transplante do cravo-dedefunto e do manjericão ocorreu quando as mudas atingiram cerca de $10 \mathrm{~cm}$ de altura e apresentavam de quatro a cinco folhas definitivas, aos 25 dias após a semeadura (DAS) e o transplante da fáfia foi quando apresentavam cerca de $15 \mathrm{~cm}$, aos 180 DAS.

A área de cultivo foi preparada utilizando-se uma aração e uma gradagem niveladora, com posterior levantamento dos canteiros com rotoencanteirador. A cama-de-frango foi distribuída a lanço e incorporada ao solo, a uma profundidade de 0-20 cm, um dia antes do transplante, nas parcelas correspondentes ao tratamento. Não houve necessidade de se fazer calagem, conforme a análise de solo.

Para o transplante nos canteiros, a fáfia foi arranjada em fileiras duplas, tanto no monocultivo (Figura 1A) quanto no policultivo (Figura 1C), com espaçamentos de $0,33 \mathrm{~m}$ entre fileiras simples e consequente espaçamento de 1,167 m entre fileiras duplas. O espaçamento entre plantas dentro das fileiras foi de $0,50 \mathrm{~m}$. O cravo-de-defunto e o manjericão foram arranjados em fileiras

\begin{tabular}{|c|c|c|}
\hline $0,33 \mathrm{~m} 0,33 \mathrm{~m} \mathrm{0}, 33 \mathrm{~m}$ & $0,160,33 \mathrm{~m} 0,33 \mathrm{~m} 0,16$ & 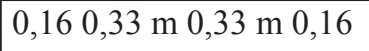 \\
\hline$\ldots F \ldots \ldots \ldots \ldots$ & 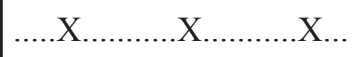 & 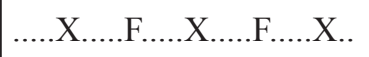 \\
\hline …...................... & 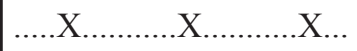 & 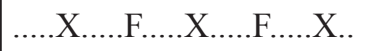 \\
\hline$\ldots . \mathrm{F} \ldots \ldots \ldots \ldots . . . .$. & 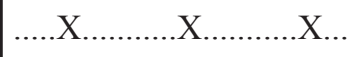 & ....X.....F....X....F.....X.. \\
\hline ....F............. & 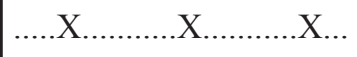 & 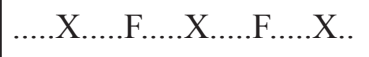 \\
\hline $\begin{array}{c}\text { Fáfia solteira } \\
\text { (A) }\end{array}$ & $\begin{array}{l}\text { Cravo de defunto ou } \\
\text { Manjericão solteiros } \\
\text { (B) }\end{array}$ & $\begin{array}{l}\text { Fáfia consorciada com } \\
\text { cravo de defunto ou man- } \\
\text { jericão }\end{array}$ \\
\hline
\end{tabular}

Figura 1. Arranjo das plantas de fáfia no monocultivo (A), manjericão ou cravo-de-defunto no monocultivo (B) e policultivo (C) (arrangement of plants of Pfaffia glomerata in monocropping (A), basil or marigold in monocropping (B) and intercropping (C). Dourados, UFGD, 2005/2006.

$0,33 \mathrm{~m}=$ espaçamento entre fileiras de plantas ou do início da parcela até a fileira de plantas (spacing between rows of plants or between the beginning of the plot and the row of plants); $0,16=$ espaçamento de $0,165 \mathrm{~m}$ do início da parcela até a fileira de plantas (spacing of 0.165 $\mathrm{m}$ from beginning of the plot up to the plant row). triplas, tanto no monocultivo (Figura 1B) quanto no policultivo com a fáfia (Figura 1C), com espaçamentos de 0,33 $\mathrm{m}$ entre fileiras simples e $0,83 \mathrm{~m}$ entre fileiras triplas. Os espaçamentos entre plantas dentro das fileiras foram de 20 $\mathrm{cm}$ para o cravo-de-defunto e $40 \mathrm{~cm}$ para o manjericão.

Durante a condução do experimento, as irrigações foram feitas utilizando-se o sistema de aspersão, com o intuito de manter o solo sempre úmido, sem exata determinação quantitativa, sendo que na fase inicial, até as plantas de fáfia apresentarem em torno de $30 \mathrm{~cm}$ de altura, o que ocorreu aproximadamente aos 45 dias após o transplante, os turnos de rega foram diários e, posteriormente, a cada dois dias. O controle de plantas daninhas foi de forma manual entre as fileiras e entre as plantas e com o auxílio de enxada entre os canteiros.

Foi avaliada a altura de plantas de fáfia a partir do transplante das mudas, até os 360 dias após, em intervalos de 30 dias, utilizando-se régua de madeira graduada em centímetros, colocada desde o nível do solo até a inflexão da folha mais alta. Na colheita aos 365 dias após o transplante, as plantas foram arrancadas inteiras e feita a separação das partes aéreas e raízes. Foi determinado o número de raízes por planta e, com o auxílio de um paquímetro digital, mediu-se o diâmetro de uma amostra de dez raízes. Todas as partes das plantas foram pesadas, em balança digital com precisão de 0,01 g para obtenção da massa fresca. Posteriormente, foram cortadas, colocadas em sacos de papel e acondicionadas em estufa com circulação de ar forçada, com temperatura média de $60 \pm 2^{\circ} \mathrm{C}$ até massa constante, para obtenção das massas secas. Nas massas secas das raízes e das partes aéreas foram avaliados os teores de nitrogênio $(\mathrm{N})$, fósforo $(\mathrm{P})$ e potássio $(\mathrm{K})$, pelos métodos semi-micro-Kjeldahl, colorimétrico do metavanadato molibdato e fotometria de chama de emissão através do Fotômetro de chama, respectivamente, seguindo a metodologia descrita por Malavolta et al. (1997).

A avaliação da produção dos capítulos florais do cravo-de-defunto foi feita colhendo-os em todas as plantas, pela manhã, usando como índice de colheita 
a abertura das flores liguladas. As colheitas foram feitas a partir de 60 dias após o transplante (DAT), com intervalo médio de seis dias, durante dez semanas consecutivas. Para obtenção de massas frescas e secas das partes aéreas de todas as plantas, as plantas foram cortadas rente ao solo, quando havia sinais de senescência de cerca de $50 \%$ das folhas, aos 130 DAT.

Para o manjericão, foram feitas duas colheitas, cortando-se as partes aéreas, a $10 \mathrm{~cm}$ do nível do solo, sendo a primeira no início do florescimento, aos 80 DAT, e a segunda, após a rebrota, aos 150 DAT, em pleno florescimento. Para a obtenção das massas frescas e secas da parte aérea total foi considerada a soma das duas colheitas.

O policultivo foi avaliado utilizando a expressão da razão de área equivalente (RAE) proposta por Caetano et al. (1999), a saber: $\mathrm{RAE}=\mathrm{Fp} / \mathrm{Fm}+\mathrm{Cp} / \mathrm{Cm}$ $+\mathrm{Mp} / \mathrm{Mm}$, onde respectivamente, $\mathrm{Fp}$, $\mathrm{Cp}$ e $\mathrm{Mp}=$ produções de massa fresca da fáfia, do cravo-de-defunto e do manjericão em policultivo e Fm, Cm e Mm = produções de massa fresca da fáfia, do cravo-de-defunto e do manjericão em monocultivo.
Aos dados de altura das plantas da fáfia foram ajustadas equações de regressão, em função dos dias após o transplante, com o emprego de polinômios ortogonais. Os dados de produção foram analisados individualmente por espécie e submetidos à análise de variância, utilizando-se o aplicativo computacional SAEG. Quando detectou-se significância pelo teste $\mathrm{F}$, as médias de produção para as formas de cultivo de fáfia foram testadas por Tukey, até $5 \%$ de probabilidade.

\section{RESULTADOS E DISCUSSÃO}

As alturas das plantas de fáfia apresentaram crescimento quadrático, independente da forma de cultivo (Figura 2), sendo a altura média máxima de $169 \mathrm{~cm}$, alcançada aos cerca de 300 dias após o transplante. Isso indica que as plantas apresentaram taxas de crescimento características e pouco influenciadas pelo ambiente. Os valores obtidos são semelhantes àqueles de Nascimento et al. (2007), exceto quanto à época da altura máxima, pois quando cultivaram a fáfia com a tansagem, observaram alturas máximas das plantas de fáfia, sob

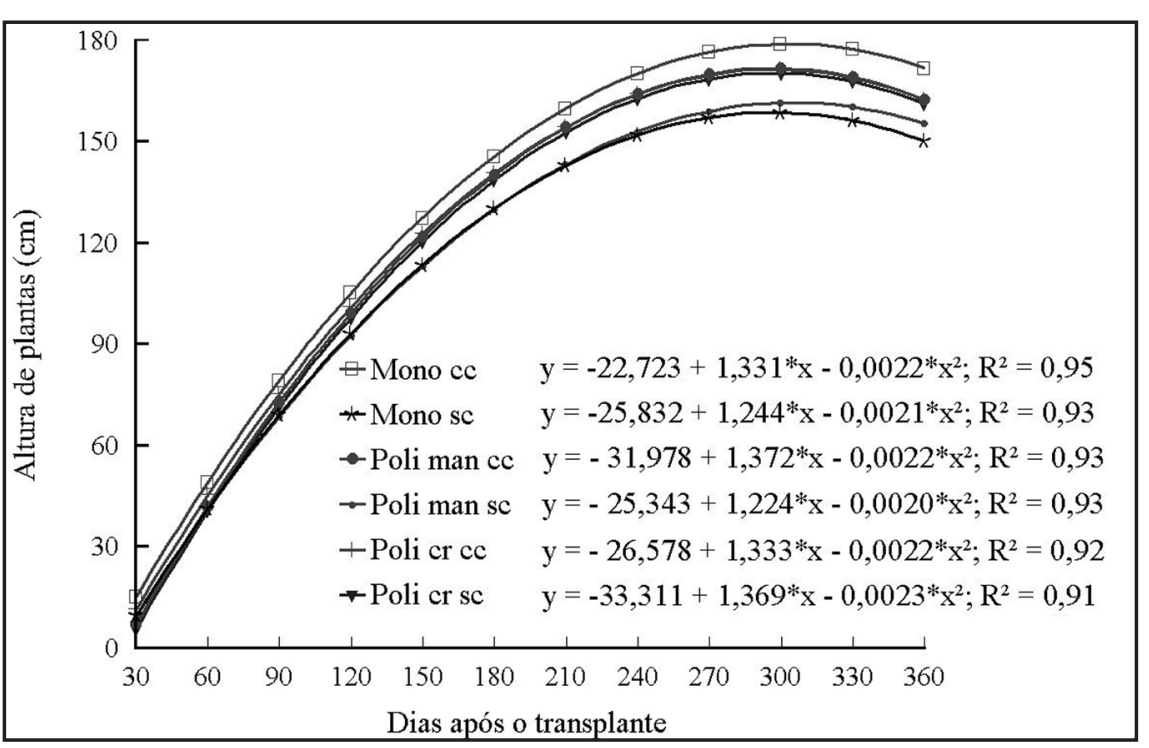

Figura 2. Altura de plantas de fáfia, em função da idade, em mono e policultivo com manjericão e cravo-de-defunto, com e sem cama-de-frango incorporada ao solo; $\mathrm{CV}(\%)=4,07$; $($ mono $=$ monocultivo $;$ poli $=$ policultivo $; \operatorname{man}=$ manjericão $; \mathrm{cr}=$ cravo-de-defunto $; \mathrm{cc}=\mathrm{com}$ cama-de-frango; $\mathrm{sc}=$ sem cama-de-frango) (height of plants of Brazilian ginseng, depending on the age, in mono and intercropping cultivation with basil and marigold, with and without poultry manure incorporated to the soil) $($ mono = monocropping; poli = intercropping; man $=$ basil; $\mathrm{cr}=$ marigold; $\mathrm{cc}=$ with poultry manure; $\mathrm{sc}=$ without poultry manure $).$ Dourados, UFGD, 2005/2006. cultivo solteiro, de $185 \mathrm{~cm}$ e $183 \mathrm{~cm}$, aos 172 e 164 dias após o transplante, nos espaçamentos entre fileiras de 54 e $36 \mathrm{~cm}$, respectivamente. As diferenças nos valores e épocas de obtenção das alturas máximas devem ser resultado de condições climáticas e de solo diferentes e ao fato de a fáfia ter caules facilmente quebradiços e que rebrotam constantemente, o que deve ter sido distinto nos dois trabalhos.

As maiores produções de massas frescas e secas da parte aérea da fáfia no monocultivo (Tabela 1) confirmam a citação de Santos (1997) de que, sob policultivo, a produção é menor. A ausência do efeito da cama-de-frango indica que o solo já tinha teor de matéria orgânica suficiente para suprir as necessidades das plantas da fáfia, conforme a análise apresentada. Resultados diferentes foram observados por Nascimento et al. (2007), quando avaliaram a capacidade produtiva da fáfia e da tansagem, em monocultivo com duas fileiras de fáfia espaçadas de 36 ou de $54 \mathrm{~cm}$, e em policultivo com três e quatro fileiras de plantas de tansagem por canteiro e observaram que o tipo de cultivo e os espaçamentos entre fileiras não influenciaram na produção de massa fresca e seca da parte aérea da fáfia.

$\mathrm{O}$ número e as massas frescas e secas das raízes da fáfia não foram influenciados pelo policultivo e nem pela cama-de-frango (Tabela 1). Esses resultados podem ser explicados por Larcher (2000) e Taiz \& Zeiger (2004) quando citam que os sistemas vegetais têm mecanismos de auto-regulação, baseandose na capacidade de adaptação do organismo individual e das populações ou no equilíbrio das relações de interferência, como competição por nutrientes, água e outros. Resultados semelhantes foram obtidos por Nascimento et al. (2007), ao constatarem que o policultivo e os espaçamentos entre fileiras no cultivo de fáfia com tansagem não influenciaram a produção de massa fresca e seca da parte aérea e das raízes da fáfia.

Os diâmetros das raízes da fáfia foram influenciados pela forma de cultivo, sendo os maiores valores obtidos nas raízes das plantas sob policultivo com manjericão e naquelas sob monocultivo. Como os diâmetros das raízes 
Tabela 1. Massas frescas (MFPA) e secas da parte aérea (MSPA), número de raízes, massas frescas (MFRA) e secas (MSRA) e diâmetro de raízes de fáfia aos 365 dias após o transplante, em mono e policultivo, com e sem cama-de-frango incorporada ao solo (fresh (MFPA) and dry weight (MSPA) of the shoot, number of roots, fresh (MFRA) and dry (MSRA) weight and diameter of roots of Brazilian ginseng, 365 days after the transplant, in mono or intercropping cultivation, with and without poultry manure incorporated to the soil). Dourados, UFGD, 2005/2006.

\begin{tabular}{|c|c|c|c|c|c|c|}
\hline & $\begin{array}{l}\text { MFPA } \\
\left(\mathrm{t} \mathrm{ha}^{-1}\right)\end{array}$ & $\begin{array}{l}\text { MSPA } \\
\left(\mathrm{t} \mathrm{ha}^{-1}\right)\end{array}$ & $\begin{array}{c}\text { Número } \\
\left(1000 \text { ha }^{-1}\right)\end{array}$ & $\begin{array}{l}\text { MFRA } \\
\left(\mathrm{t} \mathrm{ha}^{-1}\right)\end{array}$ & $\begin{array}{l}\text { MSRA } \\
\left(\mathrm{t} \mathrm{ha}^{-1}\right)\end{array}$ & $\begin{array}{c}\text { Diâmetro } \\
\text { (mm) }\end{array}$ \\
\hline \multicolumn{7}{|l|}{ Forma de cultivo } \\
\hline Monocultivo & $13,22 \mathrm{a}$ & $4,39 \mathrm{a}$ & $477,50 \mathrm{a}$ & $10,71 \mathrm{a}$ & $2,09 \mathrm{a}$ & $20,90 \mathrm{a}$ \\
\hline Policultivo fáfia e cravo-de-defunto & $9,16 \mathrm{~b}$ & $2,87 \mathrm{~b}$ & 433,33 a & $9,35 \mathrm{a}$ & $1,64 \mathrm{a}$ & $17,97 \mathrm{~b}$ \\
\hline Policultivo fáfia e manjericão & $9,20 \mathrm{~b}$ & $2,43 \mathrm{~b}$ & $342,92 \mathrm{a}$ & $9,98 \mathrm{a}$ & $2,45 \mathrm{a}$ & $23,52 \mathrm{a}$ \\
\hline \multicolumn{7}{|l|}{ Cama-de-frango } \\
\hline Com & $10,28 \mathrm{a}$ & $3,21 \mathrm{a}$ & $445,00 \mathrm{a}$ & $10,13 \mathrm{a}$ & $1,91 \mathrm{a}$ & $20,00 \mathrm{a}$ \\
\hline Sem & $10,75 \mathrm{a}$ & $3,24 \mathrm{a}$ & $390,83 \mathrm{a}$ & $9,91 \mathrm{a}$ & $2,22 \mathrm{a}$ & $21,59 \mathrm{a}$ \\
\hline $\mathrm{CV}(\%)$ & 21,14 & 27,07 & 27,58 & 31,27 & 42,16 & 15,43 \\
\hline
\end{tabular}

Médias seguidas da mesma letra nas colunas, para a forma de cultivo, não diferem estatisticamente pelo teste de Tukey e para cama-defrango, pelo teste F, até 5\% de probabilidade (values followed by the same letter in the columns, for the form of cultivation, do not differ statistically for the Tukey test and for poultry manure, for the F test, up to $5 \%$ of probability).

foram maiores e os números e massas frescas e secas foram semelhantes, é provável que os comprimentos tenham sido menores.

Os teores de nitrogênio, fósforo e potássio nas massas secas das partes aéreas e das raízes da fáfia não foram influenciados pela interação policultivo e cama-de-frango, nem pelo policultivo nem pela cama-de-frango. Os teores médios de fósforo obtidos foram de $0,636 \mathrm{mg} \mathrm{kg}^{-1}$ e $0,592 \mathrm{mg} \mathrm{kg}^{-1}$, respectivamente, para a raiz e a parte aérea, sendo maiores que os requeridos para o ótimo crescimento das plantas, que fica entre 0,1 a $0,5 \mathrm{mg} \mathrm{kg}^{-1}$, dependendo da espécie vegetal e do órgão analisado (Epstein \& Bloom, 2006). Os teores de $\mathrm{N}$ e K foram em média de $10 \mathrm{mg} \mathrm{kg}^{-1} \mathrm{e}$ de $25,67 \mathrm{mg} \mathrm{kg}^{-1}$ de massa seca da raiz, respectivamente. Segundo Epstein \& Bloom (2006), o requerimento de $\mathrm{N}$ e $\mathrm{K}$ para o ótimo crescimento das plantas está aproximadamente entre 2 e $5 \%$ na massa seca dependendo da espécie e do órgão analisado. Os dados obtidos neste experimento foram maiores do que os valores de referência necessários para o desenvolvimento normal das plantas. Quanto aos sistemas de cultivo, a não ocorrência de competição na absorção de nutrientes pelas plantas no policultivo denota que a CTC e a saturação de bases estavam dentro do ótimo requerido pela fáfia, que necessita de solos com bom teor de matéria orgânica, conforme a análise de solo apresentada.

As produções de massas frescas e secas dos capítulos florais do cravo-dedefunto foram maiores nas plantas sob policultivo cravo-de-defunto e fáfia e nas plantas cultivadas em solo com cama-de-frango (Tabela 2). A maior produção das plantas sob policultivo em relação ao monocultivo mostra coerência com o trabalho de Santos (1997), sobre as plantas poderem apresentar mecanismos de compensação da produtividade, em função de modificações das populações delas nas associações, bem como nos arranjos espaciais ou mesmo em função do sincronismo de plantio e do desenvolvimento temporal das espécies. Além disso, o sombreamento da fáfia no cravo pode ter favorecido a maior produtividade de capítulos florais. O efeito positivo com o uso da cama-

Tabela 2. Número (NCF), massas fresca e seca de capítulos florais (MFCF; MSCF) e massas fresca (MFPA) e seca da parte aérea (MSPA) do cravo-de-defunto, em mono e policultivo com a fáfia, com e sem cama-de-frango incorporada ao solo (number (NCF), fresh (MFCF) and dry (MSCF) weight of floral capitula and fresh (MFPA) and dry (MSPA) weight of the shoot of the marigold, in mono and intercropping cultivation with the Brazilian ginseng, with and without poultry manure incorporated to the soil). Dourados, UFGD, $2005 / 2006$.

\begin{tabular}{|c|c|c|c|c|c|}
\hline & $\begin{array}{c}\text { NCF } \\
\left(1000 \mathrm{ha}^{-1}\right) \\
\end{array}$ & $\begin{array}{l}\text { MFCF } \\
\left(\mathrm{t} \mathrm{ha}^{-1}\right) \\
\end{array}$ & $\begin{array}{l}\text { MSCF } \\
\left(\mathrm{t} \mathrm{ha}^{-1}\right) \\
\end{array}$ & $\begin{array}{l}\text { MFPA } \\
\left(\mathrm{t} \mathrm{ha}^{-1}\right) \\
\end{array}$ & $\begin{array}{l}\text { MSPA } \\
\left(\mathrm{t} \mathrm{ha}^{-1}\right) \\
\end{array}$ \\
\hline \multicolumn{6}{|l|}{ Forma de cultivo } \\
\hline Monocultivo & $2,46 \mathrm{a}$ & $11,94 \mathrm{~b}$ & $1,09 \mathrm{~b}$ & $25,45 \mathrm{a}$ & $8,11 \mathrm{a}$ \\
\hline Policultivo cravo-de-defunto e fáfia & $2,73 \mathrm{a}$ & $14,28 \mathrm{a}$ & $1,28 \mathrm{a}$ & $25,86 \mathrm{a}$ & $9,29 \mathrm{a}$ \\
\hline \multicolumn{6}{|l|}{ Cama-de-frango } \\
\hline Com & $2,63 \mathrm{a}$ & $14,17 \mathrm{a}$ & $1,27 \mathrm{a}$ & $28,02 \mathrm{a}$ & $9,94 \mathrm{a}$ \\
\hline Sem & $2,62 \mathrm{a}$ & $12,05 \mathrm{~b}$ & $1,09 \mathrm{~b}$ & $23,33 \mathrm{~b}$ & $7,47 \mathrm{~b}$ \\
\hline $\mathrm{CV}(\%)$ & 23,77 & 9,50 & 13,51 & 18,97 & 21,21 \\
\hline
\end{tabular}

Médias seguidas da mesma letra nas colunas, não diferem entre si pelo teste $\mathrm{F}$, até $5 \%$ de probabilidade (averages followed by the same letter in the columns, do not differ for the F test, up to $5 \%$ of probability). 
Tabela 3. Massas fresca (MFPA) e seca (MSPA) da parte aérea do manjericão em mono e policultivo com a fáfia, com e sem cama-de-frango incorporada ao solo, aos 150 DAT (dias após o transplante) (fresh (MFPA) and dry (MSPA) weight of the shoot of the basil in mono and intercropping cultivation with the Brazilian ginseng, with and without poultry manure incorporated to the soil, 150 DAT (days after the transplant)). Dourados, UFGD, 2005/2006.

\begin{tabular}{lcc}
\hline & MFPA $\left(\mathbf{t ~ h a} \mathbf{~}^{-1}\right)$ & MSPA $\left(\mathbf{t ~ h a} \mathbf{~ a}^{-1}\right)$ \\
\hline Forma de cultivo & & \\
\hline Monocultivo & $34,91 \mathrm{~b}$ & $3,43 \mathrm{~b}$ \\
Policultivo manjericão e fáfia & $52,91 \mathrm{a}$ & $4,59 \mathrm{a}$ \\
\hline Cama-de-frango & & \\
\hline Com & $28,87 \mathrm{a}$ & $2,89 \mathrm{a}$ \\
Sem & $26,43 \mathrm{a}$ & $2,99 \mathrm{a}$ \\
\hline CV $(\%)$ & 37,05 & 42,57 \\
\hline
\end{tabular}

Médias seguidas da mesma letra nas colunas, não diferem entre si pelo teste $\mathrm{F}$, até $5 \%$ de probabilidade (values followed by the same letter in the columns, do not differ for the $\mathrm{F}$ test, up to $5 \%$ of probability).

de-frango incorporada ao solo, segundo Silva \& Mendonça (2007), talvez seja devido ao provável aumento de macro e micronutrientes disponíveis no solo para as plantas, redução do alumínio trocável e da fixação do fosfato, onde a matéria orgânica do solo libera parte do $\mathrm{N}$ e $\mathrm{P}$, promovendo incrementos na produção conforme a análise da cama-de-frango apresentada.

As produções de massas frescas e secas da parte aérea e dos capítulos florais do cravo-de-defunto foram influenciadas, significativamente, pelo uso da cama-de-frango, incorporada ao solo (Tabela 2). Provavelmente, a cama-defrango utilizada pode ter contribuído na regulação da temperatura e na manutenção da umidade do solo, além de ter reduzido a perda de nutrientes por lixiviação e melhorado os atributos físicos, químicos e microbiológicos do solo (Kiehl, 2008). Resultados semelhantes foram observados por Vieira et al. (2006) quando estudaram o policultivo de capuchinha com alface, sem e com incorporação ao solo de cama-defrango, pois observaram que a produção de massa fresca das flores da capuchinha não foi influenciada pelo policultivo, mas, sim pelo uso de $10 \mathrm{t} \mathrm{ha}^{-1}$ de camade-frango incorporada ao solo.

As produções das massas frescas e secas da parte aérea do manjericão não foram influenciadas pelo uso da camade-frango incorporada ao solo (Tabela 3), tal como ocorreu com a produção da fáfia, o que deve-se, provavelmente, ao fato de o solo já possuir teor de matéria orgânica suficiente para suprir as necessidades das plantas, conforme a análise apresentada. Resultados diferentes foram obtidos por Blank et al. (2005) em Ocimum basilicum L. cv. Genovese. Os autores estudaram cinco tipos de adubação (adubo formulado Hortosafra ${ }^{\circledR}$, esterco de galinha, esterco de galinha + Hortosafra $^{\circledR}$, esterco bovino, esterco bovino + Hortosafra $^{\circledR}$ ) e três horários de colheita $(8: 00,12: 00$ e 16:00 h) e observaram que a massa seca da parte aérea das plantas foi significativamente maior quando foram adubadas com Hortosafra $^{\circledR}$ e esterco de galinha, em relação ao esterco bovino. Os autores atribuíram esse resultado ao fato de o esterco bovino possuir menores teores de fósforo, potássio, enxofre e zinco. Por outro lado, as produções do manjericão foram maiores sob policultivo com a fáfia. Isso provavelmente tenha ocorrido devido à menor disponibilidade de radiação fotossinteticamente ativa para as folhas localizadas na parte inferior das plantas do manjericão, acarretando aumento no sombreamento, fazendo com que houvesse um aumento na expansão foliar e no crescimento da parte aérea para melhor interceptação solar, fenômeno denominado "evitação à sombra". Além disso, pode ter ocorrido consequente diminuição da temperatura no microclima (Larcher, 2000; Taiz \& Zeiger, 2004), o que possivelmente, pode ter favorecido o desenvolvimento do manjericão.

Considerando-se os valores das RAEs do policultivo da fáfia com o cravo-de-defunto com cama-de-frango $(2,15)$ e sem $(1,99)$ cama-de-frango, e da fáfia com manjericão com cama-defrango $(2,44)$ e sem $(3,08)$, e por eles terem sido superiores a 1,0 , concluiuse que os policultivos entre a fáfia e o cravo-de-defunto, e da fáfia com manjericão, com e sem cama-de-frango, foram efetivos. Em relação ao policultivo da fáfia com o cravo-de-defunto, pode-se ter o efeito adicional de controle dos fitonematóides presentes no solo, cuja suscetibilidade é notória nas raízes das plantas da fáfia, prejudicando o valor comercial delas (Kanno, 2006). Embora não tenha sido avaliado estatisticamente neste trabalho, observou-se visualmente, que as plantas no policultivo estavam livres de galhas.

Nas condições em que foi conduzido o experimento concluiu-se que o comportamento produtivo da fáfia foi semelhante em solo com e sem adição de cama-de-frango; os policultivos da fáfia com o cravo-de-defunto e com o manjericão foram viáveis, em solo sem e com incorporação de cama-de-frango e o melhor policultivo foi o de fáfia e manjericão, com as plantas cultivadas em solo sem adição de cama-de-frango.

\section{REFERÊNCIAS}

BENTES LB; HIDALGO AF; SILVA JF. 2000. Produção e crescimento de raízes de Pfaffia glomerata sob cultivo orgânico. In: SIMPÓSIO DE PLANTAS MEDICINAIS DO BRASIL, 16. Resumos... Recife: Universidade Federal de Pernambuco. p. 75. Resumo AG-033.

BLANK AF; SILVA PA; ARRIGONI-BLANK MF; SILVA-MANN R; BARRETOS MCV. 2005. Influência da adubação orgânica e mineral no cultivo de manjericão cv. Genovese. Revista Ciência Agronômica 36: 175-180.

CAETANO LCS; FERREIRA JM; ARAÚJO M. 1999. Produtividade da alface e cenoura em sistema de consorciação. Horticultura Brasileira 17: 143-146.

CARVALHO FILHO JLS; BLANK AF; ALVES PB; EHLERT PAD; MELOAS; CAVALCANTI SCH; ARRIGONI-BLANK MF; SILVAMANN R. 2006. Influence of the harvesting time, temperature and drying period on basil (Ocimum basilicum L.) essential oil. Revista Brasileira de Farmacognosia 16: 24-30.

CORRÊAJÚNIOR C; MING LC. 2001. Collection of fafia [Pfaffia glomerata (Spreng.) Pedersen] in north-western State of Paraná - Brazil. Acta Horticulturae 576: 29-32.

CORRÊA JÚNIOR C; MING LC. 2004. Fáfia [Pfaffia glomerata (Spreng.) Pedersen]: o ginseng brasileiro. In: ALEXIADES MN; SHANLEY P. (eds). Productos forestales, 
medios de subsistencia y conservación: estudios de caso sobre sistemas de manejo de productos forestales no maderables. Indonésia: CIFOR.

EPSTEIN E; BLOOM AJ. 2006. Nutrição mineral de plantas: princípios e perspectivas. Londrina: Editora Planta. 401 p.

FERNANDES PC; FACANALI R; TEIXEIRA JPF; FURLANI PR; MARQUES MOM. 2004. Cultivo de manjericão em hidroponia e em diferentes substratos sob ambiente protegido. Horticultura Brasileira 22: 260-264.

FREITAS CS; BAGGIO CH; SILVA-SANTOS JE; RIECK L; SANTOS CAM; JÚNIOR CC; MING LX; CORTEZ DAG; MARQUES MCA. 2004. Involvement of nitric oxide in the gastroprotective effects of an aqueous extract of Pfaffia glomerata (Spreng) Pedersen, Amaranthaceae, in rats. Life Sciences 74: 1167-1179.

FREITAS CS; BAGGIO CH; RAÚJO SL; MARQUES MCA. 2008. Effects of Pfaffia glomerata (Spreng) Pedersen aqueous extract on healing acetic acid-induced ulcers. Brazilian Archives of Biology and Technology 51: 679-683.

FREITAS CS; BAGGIO CH; TWARDOWSCHY A; SANTOS AC; MAYERA B; LUIZ AP; SANTOS CAM; MARQUES MCA; SANTOS ARS. 2009. Involvement of glutamate and cytokine pathways on antinociceptive effect of Pfaffia glomerata in mice. Journal of Ethnopharmacology 122: 468-472.

GÓMEZ-RODRIGUEZ O; ZAVALETAMEJÍA E; GONZÁLEZ-HERNANDEZ VA; LIVERA-MUÑOZ M; CÁRDENASSORIANO E. 2007. Adaptaciones fisiológicas y morfológicas en tomate asociado con Tagetes erecta y Amaranthus hypochondriacus. Revista Fitotecnia Mexicana 30: 421-428.

GUTIERREZ PRM; HERNANDEZ L; HELIODORO; HERNANDEZ GS. 2006. Antioxidant activity of Tagetes erecta L. essential oil. Journal of the Chilean Chemical Society 51: 883-886.

KAMADA T; PICOLI EAT; ALFENAS AC;
CRUZ CD; VIEIRA RF; OTONI WC. 2009. Diversidade genética de populações naturais de Pfaffia glomerata (Spreng.) Pedersen estimada por marcadores RAPD. Acta Scientiarum Agronomy 31: 403-409.

KANNO PT. 2006. Plantas medicinais alternativas de renda e com perspectiva de comércio no mercado internacional. In: WORKSHOP DE PLANTAS MEDICINAIS DE MS, 9. Palestras...Dourados: UFGD (CD ROM).

KIEHL EJ. 2008. Adubação orgânica - 500 perguntas e respostas. 2. ed. Piracicaba: Degaspari. 227 p.

LARCHER W. 2000. Ecofisisologia vegetal. São Carlos: Rima-Artes e Textos. 531 p.

LUZ JMQ; MORAIS TPS; BLANK AF; SODRÉ ACB; OLIVEIRA GS. 2009. Teor, rendimento e composição química do óleo essencial de manjericão sob doses de cama de frango. Horticultura Brasileira 27: 349-353.

MALAVOLTA E; VITTI CG; OLIVEIRA SA. 1997. Avaliação do estado nutricional das plantas: princípios e aplicações. Piracicaba: POTAFÓS. 319 p.

MARQUES LC; GALVÃO SMP; ESPINOLAE; DIAS RF; MENEZES MGM; CARLINI ELA. 2004. Psychopharmacological assessment of Pfaffia glomerata roots (extract BNT-08) in rodents. Phytotherapy Research 18: 566572.

MEDINA AL; BeMILLER JN; JANICK J; SIMON JE.1993. Marigold flower meal as a source of an emulsifying gum. New crops exploration: research and commercialization, Indianapolis, Indiana.

MENDES FR; CARLINI EA. 2007. Brazilian plants as possible adaptogens: An ethnopharmacological survey of books edited in Brazil. Journal of Ethnopharmacology 109: 493-500.

NASCIMENTO EX; MOTA JH; VIEIRA MC; HEREDIA NAZ. 2007. Produção de biomassa de Pfaffia glomerata (Spreng.) Pedersen e Plantago major L. em cultivo solteiro e consorciado. Ciência e Agrotecnologia 31:
724-730.

NETO AG; COSTA JMLC; BELATIA CC; VINHOLIS AHC; POSSEBOM LS; SILVA FILHO AA; CUNHA WR; CARVALHO JCT; BASTOS JK; SILVA MLA. 2005. Analgesic and anti-inflammatory activity of a crude root extract of Pfaffia glomerata (Spreng) Pedersen. Journal of Ethnopharmacology 96: 87-91.

OTOFUJI GM; BAGGIO CH; FREITAS CS; VELA SM; MARQUES MCA. 2005. Extrato hidroalcoólico $70 \%$ e fração emulsão das raízes da Pfaffia glomerata reduzem a secreção ácida gástrica via óxido nítrico e inibição da $\mathrm{H}^{+} / \mathrm{K}^{+}$ATPase. In: REUNIÃO ANUAL DA FEDERAÇÃO DE SOCIEDADES DE BIOLOGIA EXPERIMENTAL, XX. Anais... São Paulo: FESBE.

PERES FSC; FERNANDESAO; SILVEIRA LCP; SILVA CSB. 2009. Cravo-de-defunto como planta atrativa para tripes em cultivo protegido de melão orgânico. Bragantia 68: 953-960.

RUSSOWSKI D; NICOLOSO FT. 2003. Nitrogênio e fósforo no crescimento de plantas de ginseng brasileiro [Pfaffia glomerata(Spreng.) Pedersen] cultivadas in vitro. Ciência Rural 33: 57-63.

SANTOS RHS. 1997. Interações interespecificas em consórcio de olerícolas. Viçosa: UFV. 129 $\mathrm{p}$ (Tese doutorado).

SILVA IR; MENDONÇA ES. 2007. Matéria orgânica do solo. In: NOVAIS RF; ALVAREZ VH; BARROS NF; FONTES RLF; CANTARUTTI RB; NEVES JCL. (ed). Fertilidade do solo. Viçosa: MG, SBCS. $1017 \mathrm{p}$.

TAIZ L; ZEIGER E. 2004. Fisiologia vegetal. Porto Alegre: Artmed. 719p.

VIEIRA MC; HEREDIA ZÁRATE NA; SOUSA TM; MOTA JH; SILVA CB; MOREIRA DG; CARVALHO GP. 2006. Produção de biomassa de capuchinha em cultivo solteiro e consorciado com alface, com ou sem cobertura do solo com cama-defrango. In: CONGRESSO BRASILEIRO DE OLERICULTURA, 46. Goiânia. Horticultura Brasileira 24: 2834-2837. (CD ROM). 